\title{
Soliton transmission through a disordered system
}

A. S. Kovalev, J. E. Prilepsky, S. A. Gredeskul, and S. A. Derevyanko

Citation: Low Temperature Physics 34, 559 (2008); doi: 10.1063/1.2957008

View online: https://doi.org/10.1063/1.2957008

View Table of Contents: http://aip.scitation.org/toc/ltp/34/7

Published by the American Institute of Physics

LOW TEMPERATURE TECHNIQUES

OPTा|CAL CAVI『Y PHVSICS

MITIGATING THERMAL

\& VIBRATIONAL NOISE

DOWNLOAD THE WHITE PAPER 


\title{
Soliton transmission through a disordered system
}

\author{
A. S. Kovalev a) and J. E. Prilepsky \\ B. Verkin Institute for Low Temperature Physics and Engineering of the National Academy of Sciences of \\ Ukraine, 47 Lenin Ave., Kharkov 61103, Ukraine
}

\section{S. A. Gredeskul}

Department of Physics, Ben Gurion University of Negev, P.O. Box 653, Beer Sheva 84105, Israel

S. A. Derevyanko ${ }^{\text {b) }}$

Photonics Research Group, Aston University, Aston Triangle, Birmingham, B4 7ET, UK

(Submitted March 7, 2008)

Fiz. Nizk. Temp. 34, 707-712 (July 2008)

An exact formula for the transmission time in a disordered nonlinear soliton-bearing classical one-dimensional system is obtained. (C) 2008 American Institute of Physics.

[DOI: 10.1063/1.2957008]

\section{INTRODUCTION}

Transmission experiments are very powerful tools for probing various excitations of solid state systems. In linear ordered systems, they enable one to restore the band structure of elementary excitations (electrons, phonons, magnons etc.). In disordered systems, they permit investigation of the Anderson localization of these excitations. One of the simplest ways of incorporating disorder is to introduce into the system some kind of short-range (point-like) defects. If the wavelength of the excitation, is much larger than the average distance between defects, the disorder can be treated in the continuous limit. Here the dynamics of the system can be described with the help of some kind of macroscopic approach. In the opposite limiting case, the excitations behave mostly as bare ones between collisions with defects. The defects manifest themselves as point scattering centers only.

The situation in the nonlinear system is rather similar. The crucial difference is that here the bare elementary excitations of its linear prototype may form bound states (envelope solitons). Now in the disordered case we are dealing with soliton transmission through a disordered segment, or piece of layer. In practical applications, one mostly deals with the case where an excited soliton pulse is being transmitted through a medium with random point defects. ${ }^{1-6}$ However, here the characteristic length of the bare excitation is not the wavelength but the soliton spatial size. Therefore the two solvable limits mentioned in the previous paragraph, (i) the spatial size of the soliton is much larger than average distance between defects ${ }^{3}$ (large density of the defects), or (ii) where the size of soliton is much more smaller then this distance.

This paper is devoted to the second case (small density of the defects). Here the soliton scattering on a single defect leads to the modification of soliton parameters (the problem was partly solved in Ref. 2). But the shifts of the parameters cannot be observed experimentally. The quantities which can be observed are the total change of soliton energy and the shift of its position (or the shift of the corresponding propagation time) after transmission of the soliton through a macroscopic region of the disordered medium. Their study is very topical for information transmission through the optical fibers. ${ }^{7}$ Because of the random distribution of the defects, all these quantities are random. Therefore the subjects of interest are their probabilistic characteristics (mean values, various moments, probability densities and so on).

In this paper we study the shift of the soliton transmission time in the framework of the one-dimensional nonlinear Schrödinger equation (NLSE). In homogeneous (ordered) systems, this equation is completely integrable and possesses stable robust soliton solutions. ${ }^{8,9}$ The NLSE describes many problems of solid state physics: nonlinear magnetization dynamics in ferromagnets with easy-axis anisotropy, ${ }^{10}$ soliton motion along a quasi-one-dimensional molecular chain, the dynamics of the envelope of phonon excitations produced by intense pulsed pumping of a crystal, ${ }^{11}$ etc. Here we obtain, to second order in the weak scattering intensity, the main value and the variance of the transmission time shift through a disordered segment.

The structure of the paper is as follows. In Sec. II next we describe the model discussed and introduce all the notation needed. Section III is devoted to the soliton scattering on a single defect. The two first moments of the transmission time shift are calculated in Sec. IV. In the Conclusion we summarize the results obtained.

\section{THE MODEL}

The model under consideration is described by the nonuniform NLSE

$$
i u_{t}+u_{x x}+2|u|^{2} u=u \varepsilon \sum_{k=1}^{n} \delta\left(x-x_{k}\right), \quad(0 \leqslant x \leqslant L) .
$$

Here $u(x, t)$ is the field variable, which may have a number of physical meanings (e.g., the spin-wave density) and the subscripts denote the partial derivatives with respect to the corresponding variables (time $t$ and coordinate $x$ ). The righthand side describes the influence of point defects with intensity $\varepsilon$, placed at the points $\left\{x_{k}\right\}, 1 \leqslant k \leqslant n, 0 \leqslant x_{n} \leqslant x_{n-1}$ $\leqslant \ldots \leqslant x_{2} \leqslant x_{1} \leqslant L$. We are interested in the case of weak 
scatterers, so $\varepsilon$ is in a sense small (this condition will be clarified later).

In what follows, we assume that the defects are independently and uniformly distributed within the segment $[0, L]$ with mean distance $l$ between the adjacent defects. This means that the number $n$ of defects on the segment $[0, L]$ is random, and the probability $p_{n}$ of finding exactly $n$ defects within a segment is

$$
p_{n}=\frac{\Lambda^{n}}{n !} \mathrm{e}^{-\Lambda}, \quad \sum_{n=0}^{\infty} p_{n}=1,
$$

where $\Lambda=L / l$ is the average number of defects on the segment. The conditional probability density for finding these $n$ defects at the points $\left\{x_{k}\right\}$ is

$$
\begin{aligned}
\rho_{n}(\{z\})= & n ! \theta\left(1-\sum_{k=1}^{n} z_{k}\right) \prod_{k=1}^{n} \theta\left(z_{k}\right)=n ! \theta\left(x_{n}\right) \prod_{k=2}^{n} \theta\left(x_{k-1}\right. \\
& \left.-x_{k}\right) \theta\left(L-x_{1}\right),
\end{aligned}
$$

where

$$
z_{k}=L^{-1}\left(x_{k-1}-x_{k}\right), \quad 2 \leqslant k \leqslant n,
$$

are the scaled distances between adjacent defects, and $z_{1}$ $=1-L^{-1} x_{1}$. The probability density (3) corresponds to a canonical ensemble (a fixed number scatterers on the segment). The probability density

$$
\rho(n,\{z\})=p_{n} \rho_{n}(\{z\})
$$

with $p_{n}$ from Eq. (2) and $\rho_{n}(\{z\})$ from Eq. (3) corresponds to a grand canonical ensemble. In this case we have an infinite line with the scatterers distributed independently along the line with density $l^{-1}$, and then we cut from this line a segment with length $L$. The number of scatterers on the segment fluctuates, and its mean number is $\Lambda$.

In what follows the symbols $\left\langle f_{n}\right\rangle$ and $\langle f\rangle$ will be used for canonical (with probability density (3)) and grand canonical (with probability density (4)) averaging of the function $f$, respectively:

$$
\begin{aligned}
& \left\langle f_{n}(\{z\})\right\rangle=\int \rho(\{z\}) f_{n}(\{z\}) d z_{1} \ldots d z_{n}, \\
& \langle f(\{z\})\rangle=\sum_{n=0}^{\infty} p_{n}\left\langle f_{n}(\{z\})\right\rangle .
\end{aligned}
$$

The variances of the averaged functions are introduced in the usual way:

$$
\delta f_{n}=\sqrt{\left\langle f_{n}^{2}(\{z\})\right\rangle /\left\langle f_{n}(\{z\})\right\rangle^{2}-1}
$$

for the canonical ensemble, and

$$
\delta f=\sqrt{\left\langle f^{2}(\{z\})\right\rangle /\langle f(\{z\})\rangle^{2}-1}
$$

for the grand canonical ensemble.

In infinite system $(-\infty<x<\infty)$ without any perturbations $(\varepsilon=0)$, the NLSE possesses the well-known fundamental four-parametric soliton solution (see, e.g., Ref. 9)

$$
u_{s}(x, t)=2 i \eta \frac{\exp \left[-2 i \xi x-i \Delta(t)-i \varphi_{0}\right]}{\cosh \left[2 \eta\left\{x-x_{0}(t)-i \varphi_{1}\right\}\right]},
$$

$$
\Delta(t)=4\left(\xi^{2}-\eta^{2}\right) t, \quad x_{0}(t)=-4 \xi t .
$$

The parameter $\eta$ determines the soliton amplitude $A_{s}=2 \eta$ and its width $\sim \eta^{-1}$, while $\xi$ determines the velocity of its envelope $V_{s}=-4 \xi$. The choice of $\eta$ and $\xi$ as the main soliton parameters is motivated by the fact that the complex value $\lambda_{s}=\xi+i \eta$ for a soliton is the eigenvalue of the linear problem associated with the NLSE. ${ }^{9}$ The parameters $\eta$ and $\xi$ are related to two integrals of motion, the number of quasiparticles bound in the soliton $N_{s}=4 \eta=\int_{-\infty}^{\infty}|u|^{2} d x$, and the soliton momentum

$$
P_{s}=8 \xi \eta=i \int_{-\infty}^{\infty} u \partial_{x} u^{*} d x=V_{s} N_{s} / 2 .
$$

We suppose that parameter $\xi$ is positive and the soliton is transmitted from right to left with a velocity $V_{s}<0$.

The soliton energy can be expressed in terms of the parameters $\xi$ and $\eta$ or in terms of the integrals of motion

$$
E_{s}=8 \xi^{2} \eta\left[1-\frac{1}{3}\left(\frac{\eta}{\xi}\right)^{2}\right]=\frac{N_{s} V_{s}^{2}}{12}-\frac{N_{s}^{3}}{12}=\frac{P_{s}^{2}}{N_{s}}-\frac{N_{s}^{3}}{12} .
$$

The first term in each of these expressions is the kinetic energy, while the second one represents the potential energy of attraction of quasi-particles bound in the soliton. The number of these quasi-particles $N_{s}$ plays the role of the soliton mass. With the help of a new parameter $\alpha=-N_{s} / V_{s}$ $=\eta / \xi>0$ we distinguish two limiting cases. The first of them $\alpha \ll 1$ is the case of a "light" soliton, the kinetic energy of which substantially exceeds the binding energy of the quasiparticles forming the soliton. In the opposite case, $\alpha \gg 1$, the potential energy is much larger than the kinetic energy. This is the "heavy" soliton case.

The other two parameters-the phases $\varphi_{0}$ and $\varphi_{1}$-are not so important in a homogeneous (ordered) system, but in the inhomogeneous case they must be taken into account.

In the next Section we shall justify the following scenario of soliton transmission through a disordered segment. We consider a soliton (5), which is ideal at $t \rightarrow \infty$ and is characterized by its energy $E_{s}$, the number of quasi-particles $N_{s}$, and its velocity $V_{s}=-\sqrt{4 E_{s} / N_{s}+N_{s}^{2} / 3}$, and is incident from the right upon the segment $[0, L]$. Passing through the first (from the right) scatterer, placed at the point $x_{1}$, the soliton changes its parameters and reaches the segment $\left[0, x_{2}\right]$ with some energy $E_{s}^{\prime}$, number of quasi-particles $N_{s}^{\prime}$, and velocity $V_{s}^{\prime}=-\sqrt{4 E_{s}^{\prime} / N_{s}^{\prime}+N_{s}^{\prime 2} / 3}$. Passing through the second scatterer, the soliton changes its parameters once more and so on. Because of change of the soliton velocity on each step, its total transmission time through the entire segment differs from that in the ideal system without scatterers. The value of this shift depends on the particular realization of our random system, i.e., on the number of scatterers $n$, their positions $\left\{x_{i}\right\}$, and their intensity $\varepsilon$. So, our first step is to study the soliton transmission through a single defect and to find the corresponding transformation $\left(N_{s}, E_{s}, V_{s}\right)$ $\rightarrow\left(N_{s}^{\prime}, E_{s}^{\prime}, V_{s}^{\prime}\right)$ of its parameters.

Here we must pay attention to the fact that in homogeneous system the NLSE has an infinite number of integrals of motion, the first of which correspond to $N, P$, and $E$. In the inhomogeneous system with defects the quantities $N$ and $E$ remain integrals of motion, but $P$ does not. That is why we 
choose the integrals $N$ and $E$ as the soliton parameters. We do not take into account other integrals of motion, and that means that we suppose that under process of soliton scattering other moving solitons and solitons bound with the defects do not appear.

\section{SOLITON SCATTERING ON A SINGLE DEFECT}

\section{A. Emission of quasi-particles}

The scattering of a soliton on a single point-like defect with small intensity $\varepsilon$, placed at the origin of an infinite system, can be considered within the framework of perturbation theory with respect to defect intensity. It has been shown ${ }^{2}$ that a soliton passes through the defect only when the condition $4 \xi^{2}>\varepsilon \eta$ is fulfilled. But in the linear approximation (to order $\varepsilon$ ) the soliton demonstrates only small $\sim \varepsilon$ and physically inessential phase shifts of $\varphi_{0}$ and $\varphi_{1}$, while the soliton velocity $V_{s}$ and its amplitude $A_{s}$ do not change at all. Their changes (or changes of $N_{s}$ and $E_{s}$ ) appear only on the second order of perturbation theory $\sim \varepsilon^{2}$, where the emission of elementary (linear) excitations is taken into account. In the case of a "rather fast" soliton $\varepsilon \eta \ll \xi^{2}$ or $\alpha \ll \xi / \varepsilon$ the velocity change due to interaction with a defect is small, and the problem can be solved analytically.

The NLSE is exactly integrable by the inverse scattering technique. ${ }^{9}$ It is natural to study the problem in terms of perturbation theory based on this technique. ${ }^{12}$ Within this approach, one deals with associated with NLSE linear problem. However, considering the soliton scattering on the defect, we should also take the continuous spectrum into account. It consists of real values $\lambda=-k / 2$ simply related to the wave number $k$ of linear waves with the dispersion relation $\omega=k^{2}$.

In the presence of linear quasi-particles and soliton the integrals of motion are modified:

$$
\begin{aligned}
& N=N_{s}+\int_{-\infty}^{\infty} n(k) d k, \\
& E=E_{s}+\int_{-\infty}^{\infty} k^{2} n(k) d k .
\end{aligned}
$$

In our case, when at $t=-\infty$ we have the pure soliton $(5), n(k)$ corresponds to the density of radiated quasi-particles at $t$ $=\infty$. For fast solitons, this value was calculated in Ref. 2:

$$
n(k)=\frac{\pi}{2^{7}} \frac{\varepsilon^{2}}{\xi^{6}} \frac{\left[\left(\frac{k}{2}+\xi\right)^{2}+\eta^{2}\right]^{2}}{\cosh ^{2}\left[\frac{\pi}{4 \eta \xi}\left(\frac{k}{2}\right)^{2}-\xi^{2}+\eta^{2}\right]} .
$$

During the interaction the soliton emits $N_{f}=\int_{-\infty}^{0} n(k)$ quasi-particles in the forward (leftward) direction, and $N_{b}$ $=\int_{0}^{+\infty} n(k)$ quasi-particles are reflected from the defect and go backward (rightward; recall that our soliton moves to the left). The total number $N_{e}$ of quasi-particles emitted by the soliton passing through the defect from right $(x>0)$ to left $(x<0)$ is

$$
N_{e}=N_{f}+N_{b}=\int_{-\infty}^{\infty} n(k) d k
$$

Correspondingly, the total energy lost by the soliton is

$$
E_{e}=E_{f}+E_{b}=\int_{-\infty}^{\infty} k^{2} n(k) d k
$$

\section{B. Change of the soliton velocity and amplitude}

Substituting expressions (9) and (10) into the conservation laws $N_{s}=N_{s}^{\prime}+N_{e}$ and $E_{s}=E_{s}^{\prime}+E_{e}$, we obtain

$$
\begin{aligned}
& N_{s}^{\prime}=N_{s}-\int_{-\infty}^{\infty} n(k) d k, \\
& E_{s}^{\prime}=E_{s}-\int_{-\infty}^{\infty} k^{2} n(k) d k .
\end{aligned}
$$

From these equations, with the help of the relation $V_{s}=$ $-\sqrt{4 E_{s} / N_{s}+N_{s}^{2} / 3}$, valid for the fundamental soliton (5), we find the change of the soliton velocity

$$
V_{s}^{\prime 2}=V_{s}^{2}-\frac{4 E_{e} / N_{s}+\left(2 N_{s} / 3-4 E_{s} / N_{s}^{2}\right) N_{e}-N_{e}^{2}+N_{e}^{3} / N_{s}}{1-N_{e} / N_{s}} .
$$

The number $N_{s}$ and energy $E_{e}$ of emitted quasi-particles have order of magnitude $\varepsilon^{2}$ and are much smaller than $N_{s}$ and $E_{s}$. Therefore this last equation to accuracy $\varepsilon^{2}$ reads

$$
V_{s}^{\prime}-V_{s} \approx-F\left(N_{e}, E_{e}, N_{s}, V_{s}\right),
$$

where

$$
F\left(N_{e}, E_{e}, N_{s}, V_{s}\right)=-\frac{N_{e} V_{s}}{2 N_{s}}+\frac{4 E_{e}+N_{e} N_{s}^{2}}{2 N_{s} V_{s}} .
$$

Equations (11), (13), and (14) generally solve the problem posed. More detailed and explicit results can be obtained in the limiting cases of light and heavy solitons. In both these cases we suppose that the "rather fast soliton" condition $\alpha$ $\ll \xi / \varepsilon$ is valid. For the light solitons this means that $\alpha \ll 1$ and $\alpha \ll|V| / \varepsilon$. In this limit the soliton is similar to a wave packet with $V \sim \varepsilon$, and in some cases we may use the analogy with the interaction of a single quasi-particle with a $\delta$-function defect. For the heavy soliton the double inequality $1 \ll \alpha \ll|V| / \varepsilon$ must be fulfilled.

We start with the case of a light soliton. Here the density $n(k)$ of emitted quasi-particles (8) is strong enough and has two pronounced peaks with widths $\Delta k \sim \eta$ centered near the points $k \approx \pm 2 \xi$. The amplitude of the right peak at $k \approx 2 \xi$ is of order $\varepsilon^{2} / \xi^{2}$ and substantially exceeds that of the left peak at $k \approx 2 \xi$, which is $\alpha^{4}$ times smaller. As a result, the main fraction of the particles is reflected from the defect $N_{b}^{l} \gg N_{f}^{l}$, and the number of emitting quasi-particles is of order $N_{e}^{l}$ $\sim \varepsilon^{2} \alpha / \xi$. More detailed calculations lead to the following results for the number of quasi-particles emitted and the total emitted energy:

$$
N_{\mathrm{e}}^{l} \approx \frac{\varepsilon^{2} \alpha}{4 \xi}=\frac{\varepsilon^{2} N_{s}^{l}}{\left(V_{s}^{l}\right)^{2}},
$$




$$
E_{s}^{l} \approx 4 \varepsilon^{2} \alpha \xi=\varepsilon^{2} N_{s}^{l} .
$$

The corresponding results for the soliton amplitude $A_{s}^{l}$ $=N_{s}^{l} / 2$ and velocity $V_{s}^{l}=-\sqrt{4 E_{s}^{l} / N_{s}^{l}+\left(N_{s}^{l}\right)^{2} / 3}$ with the same accuracy read

$$
\begin{aligned}
& A_{s}^{\prime l} \approx A_{s}^{l}\left(1-\varepsilon^{2} /\left(V_{s}^{l}\right)^{2}\right), \\
& V_{s}^{\prime l} \approx V_{s}^{l}\left(1-3 \varepsilon^{2} / 2\left(V_{s}^{l}\right)^{2}\right) .
\end{aligned}
$$

The limits of applicability of these results are $\left|V_{s}\right|$ $\gg \max \left\{\varepsilon,\left|A_{s}\right|\right\}$. We emphasize (i) that velocity transformation law for a fast, light soliton does not include the soliton amplitude, and (ii) that the soliton is slowed on passing (from right to left) through the defect: $\left|V_{s}^{\prime}\right|<\left|V_{s}^{l}\right|$. In the opposite limiting case of a heavy soliton with $\alpha \gg 1$ the density of emitted quasi-particles is exponentially small $\sim \exp (-\pi \alpha / 2)$. It is almost symmetric, with a width of order $\xi \sqrt{\alpha}$ $\sim \sqrt{A_{s}^{h}\left|V_{s}^{h}\right|}$. The number of backward emitted quasi-particles only slightly exceeds the number of those emitted forward:

$$
0<N_{b}^{h}-N_{f}^{h} \approx \frac{\varepsilon^{2} \alpha^{3}}{8 \xi} \exp (-\pi \alpha / 2) \ll N_{\mathrm{e}}^{h}
$$

where the total number of emitted particles equals

$$
N_{\mathrm{e}}^{h} \approx \frac{\varepsilon^{2} \pi \sqrt{2} \alpha^{9 / 2}}{2^{6} \xi} \exp (-\pi \varepsilon / 2) .
$$

In the same approximation the total energy of the emitted quasi-particles is positive and equal to

$$
E_{\mathrm{e}}^{h} \approx \frac{\varepsilon^{2} \pi \xi \alpha^{11 / 2}}{4} \exp (-\pi \varepsilon / 2) .
$$

For a heavy soliton the transformation laws of its amplitude and velocity look more complicated:

$$
\begin{aligned}
& A_{s}^{h^{\prime}} \approx A_{s}^{h}\left(1-\varepsilon^{2} \lambda^{h} /\left(V_{s}^{h}\right)^{2}\right), \\
& V_{s}^{h^{\prime}} \approx V_{s}^{h}-2 \varepsilon^{2} \lambda^{h}\left(A_{s}^{h} / V_{s}^{h}\right)^{2} / V_{s}^{h},
\end{aligned}
$$

where $\lambda^{h}\left(A_{s}^{h}, V_{s}^{h}\right) \approx \pi\left(A_{s}^{h} /\left|V_{s}^{h}\right|\right)^{7 / 2} \exp \left(\pi A_{s}^{h} / V_{s}^{h}\right) \ll 1$. Like the light soliton, the heavy soliton is also slowed on passage through the defect. However, its velocity transformation depends strongly on the soliton amplitude.

\section{SOLITON PASSING THROUGH A RANDOM MEDIUM}

\section{A. Amplitude and velocity}

On passing through a disordered segment, a soliton suffers changes in its amplitude and velocity. Under some assumptions these changes can be easily calculated. Suppose that the density of the defects is small, so that the average distance between the defects is much larger than the soliton size. This enables us to use the results (17)-(23) obtained for an infinite system in the previous Section. Then in considering each scattering event we neglect the quasi-particles emitted during the previous events and exclude the possibility of excitation of additional solitons. Finally, assuming that the total number of scatterers is not enormously large, $n \ll \varepsilon^{-2}$, we can perform all calculations with $\varepsilon^{2}$ accuracy. In this case the total changes of the soliton amplitude $\Delta A_{s}=\left.A_{s}\right|_{x=0}$ $-\left.A_{s}\right|_{x=L}$ and velocity $\Delta V_{s}=\left.V_{s}\right|_{x=0}-\left.V_{s}\right|_{x=L}$ are additive and do not depend on the spatial realization of the defects. For example, in passing the $k$ th scatterer the light soliton loses velocity (18) in the amount $\delta V_{s}^{k}=V_{s}^{k+1}-V_{s}^{k} \approx-3 \varepsilon^{2} / 2 V_{s}^{k}$. In the right-hand side $V_{s}^{k} \approx V_{s}^{k-1}-3 \varepsilon^{2} / 2 V_{s}^{k-1}$, but we need not take the second term into account, as it gives a correction to $\delta V_{s}^{k}$ of order $\sim \varepsilon^{4}$. After $k$ steps of this procedure we obtain $\delta V_{s}^{k} \approx-3 \varepsilon^{2} / 2 V_{s}^{1}=-3 \varepsilon^{2} / 2 V_{s}^{\text {input }}$.

For the light soliton, the total shifts of the soliton amplitude and velocity are

$$
\Delta A_{s}^{l} \approx-\varepsilon^{2} n A_{s}^{l} /\left(V_{s}^{l}\right)^{2}, \quad \Delta V_{s}^{l} \approx-3 \varepsilon^{2} n / 2 V_{s}^{l} .
$$

The corresponding shifts for the heavy soliton have the form

$$
\begin{aligned}
& \Delta V_{s}^{h} \approx-\varepsilon^{2} n A_{s}^{h} \lambda^{h} /\left(V_{s}^{h}\right)^{2}, \\
& \Delta V_{s}^{h} \approx-2 \varepsilon^{2} n\left(A_{s}^{h}\right)^{2} \lambda^{h} /\left(V_{s}^{h}\right)^{3} .
\end{aligned}
$$

All quantities entering the right-hand sides of Eqs. (24) and (25) are taken at $x=L$ and correspond to their input values.

\section{B. Transmission time}

A much more important quantity from a physical point of view is the shift of the soliton transmission time. Let $v_{1}$ $>0$ be the absolute value of the input velocity of the soliton incident from the right on the segment $[0, L]$. Then, let $v_{k}$ $>0,1 \leqslant k \leqslant k \leqslant n-1$ be the absolute value of the soliton velocity between the $(k-1)$-th and $k$-th scatterers, and $v_{n+1}$ be the output velocity of the soliton which has passed through the last, $n$th scatterer. The total transmission time in the homogeneous case equals $T^{0}=L / v_{1}$, while the transmission time through a disordered segment containing $n$ scatterers,

$$
T_{n}=\frac{L-x_{1}}{v_{1}}+\frac{x_{1}-x_{2}}{v_{2}}+\ldots+\frac{x_{n-1}-x_{n}}{v_{n}}+\frac{x_{n}}{v_{n+1}},
$$

is bigger than $T^{0}$ because of the soliton slowing after each scattering evemt, $v_{1}>v_{2}>\ldots>v_{n}>v_{n+1}$. The transmission time shift $\Delta T=T_{n}-T^{0}>0$ is a very important characteristic of the various delay lines. But this shift is nonadditive and depends strongly on the particular realization of the defects. Calculation of its statistical characteristics is a more complicated problem. Let start with the light soliton. In this case, according to Eq. (18) we have a recurrence relation for the soliton velocities within two neighboring intervals between adjacent defects:

$$
v_{k+1}=v_{k}-3 \varepsilon^{2} / 2 v_{k}, \quad 1 \leqslant k \leqslant n .
$$

The solution of this last equation in the leading approximation (taking into account only terms of order $\varepsilon^{2}$ ) has the form

$$
v_{k}=v_{1}-\zeta^{*}(k-1),
$$

where

$$
\zeta^{*}=3 \varepsilon^{2} / 2 v_{1}^{*} \ll 1 .
$$

is the main small parameter of the theory. In terms of dimensionless distances $z_{k}$ between adjacent scatterers [see Eq. (4)] the total transmission time $\Delta T_{n}$ reads

$$
\Delta T_{n}=\zeta T^{0}\left[n-\sum_{k=1}^{n}(n-k+1) z_{k}\right] .
$$


Averaging the shift $\Delta T_{n}$ and its square with the probability density (3) (for the canonical ensemble, with a fixed number $n$ ) we obtain the mean transmission time shift $\left\langle\Delta T_{n}\right\rangle$ and its variance $\delta T_{n}^{l}$ for a light soliton:

$$
\left\langle\Delta T_{n}^{l}\right\rangle=\frac{n}{2} \zeta T^{0}=\frac{3 n L \varepsilon^{2}}{4 v_{1}^{3}}, \quad \delta T_{n}^{l}=\frac{\left\langle\Delta T_{n}^{l}\right\rangle_{n}}{\sqrt{3 n}} .
$$

Smallness of the variance is ensured by having a large number of scatterers $n \gg 1$, and the transmission time shift is small if $n \zeta \ll 1$. After the next averaging over the number of scatterers we obtain for a grand canonical ensemble

$$
\left\langle\Delta T^{l}\right\rangle=\frac{\Lambda}{2} \zeta T^{0}=\frac{3 L^{2} \varepsilon^{2}}{4 l v_{1}^{3}}, \quad \delta T^{l}=\left\langle\Delta T^{l}\right\rangle \sqrt{\frac{4 l}{3 L}} .
$$

These results are qualitatively the same as those for a fixed number of defects. The difference is that here instead of $n$ we have the average number of scatterers $\Lambda=L / l$, and the numerical coefficient of the variance is different.

At first glance it seems that the same problem in the case of a heavy soliton is much more complicated, because for heavy solitons we must deal with a double recurrence, for both soliton velocity and amplitude. However, in the leading approximation $\propto \varepsilon^{2}$ the only change is the appearance of an additional multiplier $\mu$ in the recurrence (23) for the velocity:

$$
v_{k}=v_{1}-\mu \zeta(k-1), \quad \mu=\frac{4}{3} \pi\left(\frac{A_{1}}{v_{1}}\right)^{11 / 2} \exp \left(-\frac{\pi A_{1}}{v_{1}}\right) .
$$

Corresponding changes should be introduced into the final results both for a fixed number of scatterers

$$
\begin{aligned}
& \left\langle\Delta T_{n}^{h}\right\rangle=\frac{n}{2} \zeta \mu T^{0}=2 \pi \frac{n L \varepsilon^{2}}{v_{1}^{3}}\left(\frac{A_{1}}{v_{1}}\right)^{11 / 2} \exp \left(-\pi A_{1} / v_{1}\right), \\
& \delta T_{n}^{h}=\frac{\left\langle\Delta T_{n}^{h}\right\rangle_{n}}{\sqrt{3 n}},
\end{aligned}
$$

and for the grand canonical ensemble

$$
\begin{aligned}
& \left\langle\Delta T^{h}\right\rangle=\frac{\Lambda}{2} \zeta \mu T^{0}=2 \pi \frac{L^{2} \varepsilon^{2}}{l v_{1}^{3}}\left(\frac{A_{1}}{v_{1}}\right)^{11 / 2} \exp \left(-\pi A_{1} / v_{1}\right), \\
& \delta T^{h}=\left\langle\Delta T_{n}^{h}\right\rangle \sqrt{\frac{4 l}{3 L}} .
\end{aligned}
$$

In this paper we have neglected the phase shifts $\varphi_{0,1}(5)$. However, they also lead to a transmission time shift which is of order

$$
\Delta T_{n}^{\varphi} \sim \frac{n \varepsilon}{v_{1}^{3}} .
$$

Therefore the results (31) for a fixed number of scatterers are valid for a sufficiently long segment $L \gg 1 / \varepsilon$, while the results (32) for the grand canonical ensemble are valid if $L$ $\gg(n l / \varepsilon)^{-1 / 2}$. The corresponding conditions for a heavy soliton, on account of the presence of the additional small pa- rameter $\mu$ in Eqs. (34) and (35), look much stronger: $L$ $\gg(\varepsilon \mu)^{-1}$ (fixed number of scatterers) and $L \gg(n l / \varepsilon \mu)^{1 / 2}$ (grand canonical ensemble). If the inequalities mentioned above are not fulfilled, the transmission time shift is described by (36) and is related to the main contribution to the phase shifts.

\section{CONCLUSION}

The propagation of an envelope soliton through a disordered system with $\delta$-function defects has been investigated in the framework of the one-dimensional NLSE in the case when the spatial size of the soliton is much smaller than the average distance between defects. In the limiting cases of light and heavy solitons we have calculated the shifts of soliton amplitude, velocity, and transmission time after propagation through a finite segment of a disordered medium a with fixed number of disordered impurities (canonical ensemble) or with a fixed average distance between disordered defects (grand canonical ensemble).

We are grateful to M. M. Bogdan for important criticisms.

This work was partially supported by a joint scientific project No.24-02-a of NAS of Ukraine and RFBR, Israel Science Foundation (Grant \#944/05), the Royal Society International Joint Project-2007/R1 (UK), the Royal Swedish Academy of Science through a cooperation program between Sweden and the countries of the former Soviet Union and also by the Swedish Research Council and a joint FrenchUkrainian project in the framework of scientific cooperation between the NASU and the CNRS.

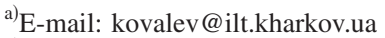

b) Also at Institute for Radiophysics and Electronics NASU, Kharkov 61085, Ukraine
}

\footnotetext{
${ }^{1}$ Yu. S. Kivshar and B. A. Malomed, Rev. Mod. Phys. 61, 763 (1989).

${ }^{2}$ Yu. S. Kivshar, A. M. Kosevich, and O. A. Chubykalo, Fiz. Nizk. Temp. 13, 438 (1987) [Sov. J. Low Temp. Phys. 13, 251 (1987)]; A. M. Kosevich, Yu. S. Kivshar, and O. A. Chubykalo, in Physics of Phonons, T. Pashkevich (ed.), Vol. 285 of Lecture Notes in Physics, Springer-Verlag (1987), p. 419.

${ }^{3}$ Yu. S. Kivshar, S. A. Gredeskul, A. Sanchez, and A. Vasquez, Phys. Rev. Lett. 64, 1693 (1990).

${ }^{4}$ R. Knapp, Physica D 85, 496 (1995).

${ }^{5}$ S. Burtsev, D. J. Kaup, and B. A. Malomed, Phys. Rev. E 52, 4474 (1995).

${ }^{6}$ A. Fratalocci and G. Assanto, Opt. Lett. 31, 1489 (2006).

${ }^{7}$ Yu. S. Kivshar and G. P. Agrawal, Optical Solitons: From Fibers to Photonic Crystals, Academic Press, San Diego (2003).

${ }^{8}$ V. E. Zakharov and A. B. Shabat, Zh. Exp. Teor. Fiz. 61, 118 (1971) [Sov. Phys. JETP 34, 62 (1971)].

${ }^{9}$ S. V. Manakov, S. P. Novikov, L. P. Pitaevskii, and V. E. Zakharov, Theory of Solitons, Colsultants Bureau, New York (1984).

${ }^{10}$ A. M. Kosevich, B. A. Ivanov, and A. S. Kovalev, Nonlinear Magnetization Waves. Dynamical and Topological Solitons, Naukova Dumka, Kiev (1983); Phys. Rep. 194, 117 (1990).

${ }^{11}$ F. D. Narayanamurty and C. M. Varma, Phys. Rev. Lett. 25, 1105 (1970).

${ }^{12}$ V. I. Karpman and E. M. Maslov, Zh. Eksp. Teor. Fiz. 73, 537 (1977) [Sov. Phys. JETP 46, 281 (1977)]; D. J. Kaup, SIAM J. Appl. Math. 31, 121 (1976); D. J. Kaup and A. C. Newell, Proc. R. Soc. London, Ser. A 361, 413 (1978).
} 